Pedagogía y Saberes No. 51

Universidad Pedagógica Nacional

Facultad de Educación. 2019. pp. 9-22

\title{
Investigar la práctica pedagógica en la formación inicial de maestros
}

Artículo de investigación

Researching the Teaching Practice

in Initial Teachers Training

Pesquisar a prática pedagógica na formação inicial de profesores

Rosa María Bolívar Osorio*

Para citar este artículo:

Bolívar, R. M. (2019). Investigar la práctica pedagógica en la formación inicial de maestros. Pedagogía y Saberes, 51,9-22.

Profesora de la Universidad de Antioquia (UdeA). Candidata a doctora del Doctorado Interinstitucional en EducaciónUniversidad el Valle e investigadora del Grupo de Historia de la Práctica pedagógica (GHPP).

Correo electrónico: rosabolivar14@gmail.com

Código ORCID: orcid.org/0000-0003-4005-0419. 


\title{
Resumen
}

Este artículo, derivado de una investigación, presenta un estado del arte de las investigaciones en el campo de la formación de maestros que estudian la práctica pedagógica y, en particular, el enfoque de práctica reflexiva. Con base en el análisis de 65 publicaciones nacionales e internacionales publicadas entre el 2000 y el 2016 se presentan los hallazgos en dos grandes categorías, práctica pedagógica y práctica reflexiva. Prevalecen las investigaciones bajo el enfoque de estudios de caso, sistematizaciones de experiencias y evaluaciones de las prácticas en los programas. En conclusión, se reconoce el alcance global del tema y la necesidad de emprender estudios de mayor envergadura sobre este problema en Colombia.

\section{Palabras clave}

práctica pedagógica; formación inicial de maestros; práctica reflexiva; practicum

\begin{abstract}
This article, derived from an investigation, presents a state of the art of research in the field of teachers training that studies the pedagogical practice and, in particular, the reflective practice approach. Based on the analysis of 65 national and international publications published between 2000 and 2016, the findings are presented in two major categories, pedagogical practice and reflective practice. Investigations prevail under the focus of case studies, systematization of experiences, and evaluations of practices in the programs. In conclusion, the global scope of the topic and the need to undertake more extensive studies on this problem in Colombia are recognized.
\end{abstract}

\section{Keywords}

Teaching practice; initial teachers training; reflective practice; practicum

\section{Resumo}

Esse artigo, derivado de uma pesquisa, apresenta um estado da arte das pesquisas no campo da formação de professores que estudam a prática pedagógica e, em particular, a abordagem da prática reflexiva. Foram revisadas 65 publicações nacionais e internacionais publicadas entre 2000-2016. As descobertas se apresentam em duas grandes categorias: prática pedagógica e prática reflexiva. Ainda que o tema tenha alcance global, observa-se que prevalecem as pesquisas como sistematizações de experiências e avaliações das práticas nos programas, quase todos como estudos de caso. Permanece a necessidade de empreender estudos mais amplos sobre este problema na Colômbia.

\section{Palavras-chave}

prática pedagógica; formação inicial de professores; prática reflexiva; practicum 


\section{Introducción ${ }^{1}$}

E n Colombia, el debate educativo actual se fundamenta en la premisa de que la calidad de la educación está relacionada de modo directo con la calidad de la formación de los maestros, de allí se deriva la creencia de que entre mayor y mejor sea dicha formación, mejor será la calidad de la educación y podremos llegar a alcanzar la meta de tener a Colombia como la más educada en el 2025. Esta idea ha permeado todas las políticas de formación de los gobiernos recientes, y así pasamos en los programas de formación de educadores de un componente práctico subordinado a la teoría, a un componente práctico que se reclama como esencial, transversal, estructurador de la formación. Los resultados de los últimos programas implementados ${ }^{2}$ interrogan la formación inicial, y de ella, entre todos los aspectos que contempla, parece existir un acuerdo entre los teóricos de la formación de maestros, los formadores, los organismos internacionales y los legisladores en que el aspecto de mayor trascendencia en dicha formación de los maestros es su práctica pedagógica y su orientación es la que los convertirá en profesionales de calidad.

Comprender esta problemática nos obliga a situarla históricamente y a revisar los discursos en los cuales se han sustentado las prácticas pedagógicas en la formación de maestros, sobre todo, cuáles hicieron posible que adquirieran ese lugar protagónico; para ello, un punto de partida es identificar el lugar de la práctica pedagógica en el contexto internacional de la formación docente, de tal manera que realizar un estado del arte de las investigaciones recientes es más que una tarea necesaria.

Una revisión general nos muestra que los procesos de práctica pedagógica y su contribución al aprendizaje de los futuros maestros se han convertido desde hace unos 30 años en un área de interés para los investigadores, educadores de maestros y maestros. Justamente, a partir de la pregunta por la formación de maestros, cómo, para qué y con qué herramientas formarlos, desde finales de los años 80 circula con fuerza en el contexto internacional el discurso acerca de la práctica reflexiva con el trabajo de Donald Schön $(1983,1987)$ quien planteó como alternativa al modelo de formación basado

1 Artículo de revisión o estado del arte derivado del proyecto de investigación doctoral La práctica pedagógica y el diagrama de la reflexión: la emergencia del discurso sobre la práctica reflexiva en la formación inicial de maestros en Colombia. Programa de Doctorado Interinstitucional en Educación. Universidad del Valle.

2 Véase por ejemplo Díaz, Barreira y Pinheiro (2015). en la racionalidad técnica, el modelo de reflexión en la acción. A partir de estos planteamientos las propuestas de formación de educadores se centran en la práctica y toman como eje central la experiencia y la reflexión para aprender de ella. Con fundamento en sus ideas, para aplicarlas, desarrollarlas, modelarlas y en menor medida para criticarlas, se ha investigado y se sigue investigando en todo el mundo. ${ }^{3}$

Este estado del arte se inserta en el campo de estudios sobre la formación de maestros, y se ocupa del componente específico denominado práctica pedagógica, que ha recibido varias denominaciones según las diferentes tradiciones de la pedagogía. En algunos casos se le nombra como praxis, práctica docente, práctica de enseñanza o práctica pedagógica; $y$ al referirse al momento en que ocurre puede aparecer en la literatura como práctica profesional, práctica académica, practicum, entre otras.

Conscientes de que estamos en un campo de conocimiento bastante amplio y complejo, esta revisión sistemática incluye los estudios sobre práctica pedagógica en el proceso de formación inicial de maestros, con énfasis en aquellos estudios que utilizan el enfoque teórico o metodológico de la práctica reflexiva. ${ }^{4}$

\section{Metodología}

Un proceso de revisión sistemática requiere una selección rigurosa con criterios explícitos. Para construir este estado del arte se revisaron 1498 registros obtenidos de la base de datos ERIC. El término de búsqueda inicial utilizado fue "teaching practice" y se aplicó el filtro "relationship theory practice" restringido a investigaciones publicadas entre los años 2000 y 2016. Para enriquecer la búsqueda de los estudios colombianos se acudió a los repositorios de tesis de la Universidad de Antioquia, Universidad San Buenaventura, Universidad de la Salle y Pontificia Universidad Javeriana; complementándola con la búsqueda orientada de referencias. Los trabajos se seleccionaron, revisaron y analizaron de acuerdo con los propósitos del estudio, sus enfoques metodológicos y las principales conclusiones a las que llegaron.

3 Este estado del arte encontró referencias a sus trabajos en países de los cinco continentes.

4 Esta denominación agrupa los diferentes estudios y propuestas teóricas y/o metodológicas que toman como fundamento la reflexión para la formación y el desarrollo profesional docente y que en la actualidad circulan principalmente gracias al esfuerzo de la Plataforma Internacional Práctica Reflexiva. Véase http://www.practicareflexiva.pro/ 
Producto de esta búsqueda se consolidó una base de datos con 190 textos a los cuales se les aplicaron los siguientes criterios que operaron como filtro definitivo:

- Ser publicado entre el 2000 y el 2016.

- Preocuparse por la práctica pedagógica en la formación de maestros.

- Estar publicados bajo el formato artículo de investigación, tesis o libros teóricos.

- Centrarse en la práctica dentro de los programas de formación inicial de maestros.

De esta manera, se seleccionaron en total 65 trabajos entre los que se encuentran artículos de investigación (43), tesis de pregrado (4), libros teóricos (10), tesis de maestría (6) y tesis de doctorado (2), como se indica en la tabla 1. Los trabajos identificados proceden de diferentes países: 23 son de Colombia y el resto proviene de Estados Unidos (8), España (4), Turquía (4), Argentina (3); también se encuentran de Brasil, Chile, Venezuela, Australia, Croacia, Nigeria, Malasia y Canadá, entre otros.

\section{Hallazgos}

\section{Práctica pedagógica}

Con base en los criterios previamente expuestos, se encontraron 40 trabajos que investigan sobre las prácticas pedagógicas de maestros en formación, y se hizo énfasis en aquellos que ponen en tensión la relación teoría-práctica. Según el objeto central del que se ocupan se han agrupado en 6 subcategorías que se explican a continuación:

\section{Historias, sistematizaciones y evaluaciones}

Sistematizar y evaluar las prácticas es el propósito más frecuente entre las investigaciones encontradas. Se destacan dos estados del arte, en primer lugar, el de Lawson, Çakmak, Gündüz y Busher (2015), quienes revisan 114 investigaciones publicadas entre el año 2000 y el 2012. El alto número de trabajos encontrados por ellos es una muestra de la relevancia de las prácticas en el contexto global; hay una prevalencia de los estudios cualitativos. Llama la atención que no encontraron estudios sobre la lectura que hacen los estudiantes de las escuelas del practicum. Según los autores, casi todos los estudios se centran solo en uno de los actores, pero no abordan la complejidad de la práctica; son limitados los estudios comparados porque cada estudio debe leerse en el contexto de su sistema educativo y cada sistema educativo es único; terminan con la invitación a realizar investigaciones que incluyan a todos los actores involucrados con el ánimo de ver the whole picture acerca de la práctica.

En segundo lugar, encontramos en Colombia a Yolima Bolaño (2014), quien en su tesis de maestría realiza un estado del arte sobre las investigaciones de prácticas pedagógicas de los maestros en formación en universidades colombianas desde el 2010 hasta el 2013. Retoma 86 tesis de cinco universidades asociadas a ASCOFADE y evidencia que en la mayoría de ellas aún se asume la práctica como un entrenamiento, por lo cual, se hace un llamado a un mayor acompañamiento "no desde la posición de supervisar al practicante sino como estrategia de un proceso de evaluación formativa que lo ayude a constituirse como un docente reflexivo en su práctica pedagógica" (p. 59).

En esta misma categoría, se destacan a nivel internacional investigaciones que con herramientas de la investigación cualitativa, en especial con el estudio de caso, quieren evaluar la efectividad de las prácticas (Cometta, Domeniconi y López, 2005; Endeley y Nalova, 2014; Göçer, 2013; Mannathoko, 2013; Yalin UÇar, 2012), todos concluyen que este periodo de prácticas no es efectivo; identifican problemas en la supervisión y duración del programa (Endeley y Nalova, 2014; Yalin UÇar, 2012), encuentran cómo la práctica mejora los conocimientos pedagógicos de los estudiantes pero no mejora su rendimiento en el saber específico (Mannathoko, 2013), y reconocen la fragmentación y superposición de contenidos (Cometta, Domeniconi et al., 2005). Otro estudio de evaluación encontrado es el de Oluwatayo y Adebule (2012) que muestra que las diferencias de género y la experiencia previa como docentes no marcan una distinción significativa en el practicum. Y, por último, el estudio de Cometta, Bentolila, Clavijo, Domeniconi y López (2005) quienes se ocupan de identificar las innovaciones en los procesos de formación de docentes.

En el contexto colombiano encontramos cuatro investigaciones que tienen el propósito de sistematizar el desarrollo de las prácticas en situaciones específicas. Arenas, Bermúdez y Fandiño (2015) recogen el trabajo de los directores de prácticas a través de la metodología de sistematización reflexiva; Herrera y Larios (2012)cuyo objetivo es determinar las estrategias que asume la Escuela Normal Superior de Cartagena de Indias (ENSCI), evalúan a través de la etnografía cómo las prácticas pedagógicas responden o no a las necesidades de formación de los estudiantes del ciclo complementario. Con una perspectiva histórica, Conde y Cortés (2006) realizan una reconstrucción de carácter descriptivo que se propone mostrar 
Tabla 1

Síntesis de material incluido

\begin{tabular}{|l|c|c|c|}
\hline \multicolumn{1}{|c|}{ Tipo de Material } & $\begin{array}{c}\text { Práctica } \\
\text { pedagógica }\end{array}$ & Práctica reflexiva & Total \\
\hline Artículos de investigación & 27 & 16 & 43 \\
\hline Tesis de Doctorado & & 2 & 2 \\
\hline Tesis de Maestría & 2 & 4 & 6 \\
\hline Tesis de Pregrado & 4 & & 4 \\
\hline Libros teóricos & 7 & 3 & 10 \\
\hline TOTAL & 40 & 25 & 65 \\
\hline
\end{tabular}

Fuente: elaboración propia.

el desarrollo histórico de las prácticas en los últimos 35 años en la Universidad de La Salle, y el artículo Genealogía de la práctica pedagógica para la formación de maestros normalistas de López Gil (2010) se propone encontrar la singularidad de la práctica, para lo cual, acude a los sentimientos de los normalistas y los maestros de práctica e indaga por la conciencia de querer ser maestro y de su vocación, en una escuela normal y su escuela anexa. Su trabajo hace un esfuerzo por problematizar algunos elementos de la formación normalista, pero se queda corto en su propósito genealógico de describir las relaciones de saber-poder que hacen parte de esta formación.

\section{Pedagogía de la práctica pedagógica}

Hay consenso entre los formadores de maestros en cuanto a que los programas deben tener un componente pedagógico; pero los debates sobre su extensión, contenido, ubicación y finalidad ocupan a muchos investigadores en la actualidad. En esta subcategoría se incluyen en el contexto internacional investigaciones que se han preguntado por los principios pedagógicos que deben tener los programas de formación de maestros (Korthagen, Loughran y Russell, 2006) y que se preocupan por cómo los estudiantes de práctica aprenden los conceptos pedagógicos (González y Gómez, 2014).

En el centro de esta categoría están los trabajos sobre la práctica y sus múltiples orientaciones; preguntas por el sentido de la asesoría en la práctica (García y Martínez, 2006), por el papel de las prácticas en la formación inicial (Gallego, Pérez, Torres y Gallego Torres, 2006) y el análisis de las racionalidades o concepciones sobre la práctica y cómo estas influyen en los procesos de formación son frecuentes, pero pocas veces van más allá de la descripción y no arriesgan propuestas teóricas que conceptualicen y transformen los planes de formación.
Se destaca en Colombia, el trabajo de la profesora Marta Lucía Quintero (2008), quien a partir de su tesis de doctorado intentó configurar la práctica como un objeto de estudio. A partir de allí se arriesga a construir una propuesta metodológica para la enseñanza de la práctica docente en la Universidad de Antioquia y luego un modelo de autoevaluación para la práctica en los programas de licenciatura (Quintero y Duque, 2010). Estas propuestas en su momento tuvieron un amplio despliegue y aún hoy hacen parte de la base y el funcionamiento de la práctica en esta institución.

\section{Representaciones de los agentes de la práctica}

Uno de los asuntos más recurrentes en las descripciones de los problemas de las prácticas pedagógicas tiene que ver con la poca articulación entre teoría y práctica que perciben los agentes participantes, en particular los estudiantes. Como ya lo habían identificado Lawson et al., (2015) no se encuentran estudios que de manera simultánea trabajen con todos los agentes involucrados en la práctica, es más común encontrar la pareja maestros practicantes-maestros formadores o maestros practicantes- maestros cooperadores. En este estado del arte encontramos trabajos que se enfocan en las percepciones - representaciones de los maestros practicantes - acerca de la práctica (Busher, Gündüz, Çakmak y Lawson, 2015; Kennedy, 2004); ejemplo de ello lo encontramos en Brasil, donde Marimar Da Silva (2005) viene indagando cómo cambian sus habilidades de enseñanza con la práctica.

En Colombia es Baquero (2006) quien analiza las racionalidades que han orientado la práctica pedagógica para explicar sus formas de organización y desarrollo, además, las repercusiones en los procesos formativos y en la formación del maestro como sujeto de saber pedagógico. 


\section{Investigación y práctica pedagógica}

Posicionada como una de las estrategias de formación de docentes, desde hace más de dos décadas la investigación en el aula se ha convertido en un tema relevante en los estudios sobre formación inicial de maestros. En este estado del arte se identificaron seis trabajos en donde la pregunta por la práctica docente está articulada a la investigación en el aula, como estrategia que ayuda a los educadores en formación a significar su experiencia.

En el contexto internacional, White Dean y Todd (2000) consideran que se logra un cambio en las prácticas cuando se empodera a los maestros en formación con las herramientas de la investigación; Vitarelli (2005) enfoca su trabajo en la función formativa y reflexiva de la investigación para la formación de maestros y Torres y Mercado (2004), basadas en el análisis de los diarios reflexivos, el diálogo entre pares y los informes de investigación de los maestros en formación, concluyen que la investigación de los maestros es un vehículo de la praxis genuina de la formación de educadores.

En Colombia, es preciso resaltar el trabajo de Gutiérrez y Buitrago (2009), ellas se ocupan de desarrollar una propuesta para la formación en las prácticas basada en la investigación lo cual permite una mayor articulación teoría-práctica; en este punto coinciden con Niño (2011), quien plantea estructurar propuestas de formación de docentes desde un currículo integrador.

Esta relación entre práctica pedagógica e investigación se ha concretado entre otras cosas en el modelo de formación en la práctica que algunos han denominado Práctica Pedagógica Investigativa. Saker García (2014), en su investigación doctoral, centra su objeto de estudio en este modelo, como experiencia que fundamenta el ejercicio docente en cuatro centros educativos del caribe colombiano; para ello referencia los postulados epistemológicos, teóricos y conceptuales, así como procedimientos, métodos, estrategias y acciones que regulan la interacción, el pensamiento, los imaginarios, las posiciones, oposiciones y discursos del objeto de formación de los estudiantes-maestros, que adelantan estudios pedagógicos en los programas de formación complementaria de las Escuelas Normales Superiores (ENS). En la misma perspectiva y como un estudio de caso, Isaza, Henao y Gómez (2005) sistematizan, evalúan y analizan la Práctica Pedagógica Investigativa en la Facultad de Educación de la Universidad de Antioquia y, a partir de la comprensión lograda, proponen una perspectiva teórica y metodológica para las prácticas en dicha facultad.

\section{Dispositivos de formación}

Otra subcategoría emergente es la que agrupa los estudios sobre dispositivos para formar en la práctica, desde metodologías diversas y con poblaciones muy delimitadas estos investigadores sistematizaron sus experiencias con dispositivos como la integración de las Tic en el practicum (Caner, 2010; Karami, Karami y Attaran, 2013; Madlela, 2015), las rondas de clase (Bowman y Herrelko, 2014), los estudios de caso (Da Graca y Mizukami, 2002), las narrativas, las autobiografías (Chavarría y Echavarría, 2014; Garvis, 2012) y el trabajo por pares (Koç, 2011).

Se aprecia que en todas las latitudes la pregunta por el cómo formar maestros es una pregunta vigente sobre la que se experimenta cada día. Estos estudios nos hablan de esa búsqueda de los educadores por encontrar nuevos dispositivos de formación con los cuales combatir ese fantasma de la tensión teoríapráctica, que hace pensar a los maestros que las herramientas adquiridas en su formación son insuficientes ante la realidad compleja del ejercicio magisterial.

\section{Relación con los centros de práctica}

Aunque en la elaboración del presente estado del arte no aparecieron muchos trabajos que consideren este aspecto, es pertinente mencionarlo porque es un campo en el que aún hay mucho por estudiar, en particular en Colombia donde las relaciones que establecen los centros formadores con los centros de prácticas resultan accidentales y circunstanciales la mayoría de las veces. Así, estos trabajos sobre las mentorías (Rakicioglu-Soylemez y Eroz-Tuga, 2014) y sobre la relación de la universidad formadora con los centros de práctica (Villegas, Londoño, Cárdenas y Mosquera, 2015) se convierten en pioneros en un campo de trabajo poco estudiado y de urgente necesidad en Colombia.

Decimos de urgente necesidad porque en este momento en el que se ha demandado a las instituciones formadoras que aumenten el número de créditos académicos dedicados a las prácticas, se incrementa la demanda por espacios para realizarlas. En nuestro país, la relación entre instituciones formadoras y centros no está reglamentada. Media entre ellas un acuerdo de voluntades que depende en gran medida del maestro que decida tener en su espacio a un maestro en formación; toda vez que por esta actividad un maestro en ejercicio no recibe ningún reconocimiento o estímulo, ni por parte del Ministerio de Educación, ni por parte de la institución formadora. Las tensiones que activa este problema siguen pendientes de ser analizadas e investigadas. 
Investigaciones que utilizan la perspectiva teórica y metodológica de la práctica reflexiva

En esta categoría se encontraron 25 trabajos que se han agrupado para su presentación en cuatro subcategorías, pues aunque a simple vista podría pensarse que tratan un marco común, hay acentuadas diferencias en los propósitos con los que abordan la perspectiva de la práctica reflexiva.

\section{Proponen marcos teóricos}

Además de los trabajos ya mencionados de Donald Schön, en esta categoría el estudio principal es el de Perrenoud (2004), titulado Desarrollar la práctica reflexiva en el oficio de enseñar. De acuerdo con este autor, la práctica reflexiva es una alternativa para profesionalizar el oficio de maestro. En su trabajo presenta la importancia de hacer de la práctica reflexiva un hábitus en el profesor; despliega su enfoque al que denomina clínico y se dirige a los formadores de maestros acerca de los desafíos que representa hacer su labor desde esta perspectiva.

Debe destacarse, además, el trabajo desarrollado en el año 2008 por Ángels Domingo Roget, como tesis de doctorado, sobre lo que ella denomina "la metodología formativa de la Práctica Reflexiva en la formación universitaria" (p. 233). Se trata de una investigación evaluativa sobre un modelo de práctica reflexiva diseñado para formación inicial de maestros. En el 2014, en conjunto con María Victoria Gómez, publicó un texto con formato de manual para todos aquellos que quieran implementar este modelo de formación (Domingo Roget y Gómez, 2014.

Pero quizás el impacto más importante del trabajo de Ángels Domingo es la creación en el año 2011 de la Plataforma Internacional Práctica Reflexiva ${ }^{5}$ concebida como una comunidad internacional que, a través de la interacción entre profesionales, expertos, y/o maestros, trabaja para "difundir, enriquecer y diversificar las metodologías formativas basadas en la reflexión orientadas a la profesionalización docente y enmarcadas con el paradigma reflexivo" (párr. 1).

5 Puede consultarse en: http://www.practicareflexiva.pro/. En la actualidad es una amplia red de profesores, investigadores de más de 52 países y preparan para este año su primer congreso internacional en la ciudad de Buenos Aires. El objetivo de la plataforma es ofrecer a personas y organizaciones: Bases conceptuales sobre el aprendizaje experiencial y reflexivo, métodos y modelos para aprender de la propia práctica, instrumentos y herramientas efectivas para el desarrollo de la reflexividad y además investigaciones y estudios actualizados para avanzar en este paradigma formativo emergente.
En Holanda se destaca el trabajo de Korthagen (2010), quien propone un enfoque de la formación basado en interpretaciones del comportamiento y aprendizaje del profesorado. Este enfoque recibe el nombre de modelo realista y se fundamenta en los resultados obtenidos en diferentes investigaciones que demuestran que gran parte del comportamiento de un profesor tiene orígenes irracionales e inconscientes, lo que limita el valor de la teoría como base para la enseñanza. Aclara por qué la reflexión es tan importante para los profesores y analiza qué aspectos son importantes para estimular la reflexión. Para Korthagen es necesaria una perspectiva más holística sobre los profesores y la enseñanza.

Desde la Universidad de los Andes en Venezuela, Villalobos y de Cabrera (2009) recogen un marco teórico acerca de la práctica reflexiva que según ellos es una necesidad de los docentes. Definen la reflexión, destacan las actitudes de los practicantes desde esta perspectiva y hacen una distinción entre niveles de reflexión - reflexión superficial, pedagógica, crítica, autorreflexión - para concluir que la práctica reflexiva requiere de mediación, acompañamiento y monitoreo constante para tener éxito.

En Colombia encontramos dos trabajos en esta categoría: el primero es de Pérez, Rodríguez y Triana (2006)quienes indagan las concepciones de profesores de educación superior acerca de lo que debe caracterizar a un profesional reflexivo, con el propósito de definir unos lineamientos curriculares para formar tales profesionales. Y en segundo lugar encontramos el trabajo de Barragán (2015) quien desde el 2004 ha estado interesado en el estudio del saber práctico del profesor con el propósito de aportar a la configuración de lo que denomina "el campo de la pedagogía hermenéutica” (p.181).

\section{Plantean la reflexión como una finalidad}

Los autores de esta subcategoría ven la reflexión como una búsqueda, como una finalidad de la formación de los maestros. Puede ser una búsqueda ética y su lugar de formación son los procesos de prácticas en la formación inicial de maestros (Villa Holguín, 2013) o la reflexión que hacen los maestros en ejercicio desde la escritura autobiográfica (Páez, Gómez y García, 2008). Desde esta perspectiva la reflexión es vista como una práctica que debe incorporarse en la rutina del maestro porque gracias a ella podrá ser un mejor maestro.

\section{Experimentan con dispositivos}

Existe un interés en la comunidad académica por encontrar diferentes dispositivos para la formación inicial de maestros que favorezcan la ya aceptada práctica reflexiva. Se sitúan aquí una amplia variedad 
de ellos: los estudios de caso (Schrittesser, 2008), el portafolio electrónico o virtual (Campaña, 2013; Lara, Pereira, Alvarado y Muñoz, 2015), los grupos reflexivos (Malkani y Allen, 2005), las narrativas (Binks, Smith, Smith y Joshi, 2009; Jiménez, 2010) y el coaching reflexivo (Rodríguez et al., 2011).

Podemos resaltar el estudio de Jones (2009), que describe la implementación del Aprendizaje Intercolegiadamente Apoyado ${ }^{6}$ y la reflexión con el modelo ALACT $^{7}$ (Korthagen, 1985, 1988). Este estudio adopta un enfoque de métodos mixtos, su propósito es encontrar maneras de aumentar la aplicación de la teoría en la práctica de los maestros en formación. Concluye que un hábito de reflexión colaborativa también apoya a los maestros al hacer conexiones entre las teorías enseñadas en la universidad y sus prácticas en el aula, con lo cual se aumenta el uso de la pedagogía basada en la investigación, la investigación de maestros y el desarrollo de la experiencia adaptativa.

\section{La práctica reflexiva como una estrategia de formación}

Es común que se asuma la práctica reflexiva como una estrategia de formación por ello esta es una de las subcategorías en la que más se encuentran estudios.

En Inglaterra, Harrison, Lawson y Wortley (2005) aplican la estrategia de la práctica reflexiva para mejorar el proceso de mentorías con maestros en formación y maestros principiantes. Parten del concepto de pensamiento crítico, pues para ellos la práctica reflexiva articula el pensamiento crítico con el aprendizaje experiencial, desarrollan un estudio cualitativo en el que observan profesores durante un año, y demuestran que la incorporación de estas estrategias cambia las formas de trabajo de estudiantes y mentores; al concluir hacen un llamado sobre la necesidad de formar a los mentores, a los directores de escuela y a los encargados de formular políticas.

Con base en las narrativas, otro grupo de estudios (Ajayi, 2011; Daniels, Pirayoff y Bessant, 2013; Joseph y Heading, 2010) utiliza los postulados de la práctica reflexiva para enriquecer los análisis sobre los textos de los maestros y destacar cómo ellos van construyendo su subjetividad de enseñantes. En su trabajo The practicum: more than practice, Schulz (2005) experimenta durante tres años cómo mejorar

6 Original en inglés Inter-collegially Supported Learning (Tigchelaar y Melief, 1997, citados en Jones, 2009).

7 El modelo de reflexión ALACT deriva su nombre de las primeras letras de las cinco fases en inglés (Action, Looking back on action, Awareness of Essentials aspects, Creating alternative methods of actions y Trial). la práctica en un programa de formación de maestros y para ello postula la investigación y la reflexión como herramientas para fortalecerla.

Cierra esta categoría el trabajo de Sagayo y Chacón (2006), en el cual se afirma que las prácticas profesionales en los planes de estudio del nivel universitario constituyen un importante referente de formación, sin embargo, se encuentran desplazadas del debate teórico. Para intentar salidas, las autoras visualizan dos rutas: en primer lugar, cambiar los enfoques de prácticas profesionales $y$, en segundo lugar, hacer de las prácticas el eje de la formación; por ello, sugieren estructurar el eje de prácticas profesionales con base en los planteamientos del enfoque hermenéuticoreflexivo, por cuanto supone su construcción como objeto de estudio y campo de intervención en la compleja situación de la cultura escolar.

\section{Discusión}

Los trabajos que hemos presentado en este estado del arte asumen posturas metodológicas diversas, no obstante, los enfoques teóricos y conceptuales no varían tanto como los metodológicos. Casi todos toman como referentes los trabajos de Dewey, Schön y Perrenoud; asumen la práctica reflexiva como el deber ser del maestro y por ende esencial en su proceso de formación. De esta manera, las investigaciones están más orientadas a evaluar cómo se ha implementado este modelo o a desarrollar y probar estrategias para poner en funcionamiento sus postulados.

Para ilustrar esta diversidad metodológica se presenta la tabla 2 que relaciona los enfoques de investigación, las herramientas de recolección de datos o técnicas de investigación y algunos ejemplos. Podrá verse que prevalecen los estudios cualitativos con una tendencia preponderante hacia los enfoques que hacen uso de las narrativas, diarios, auto estudios, biografías, autobiografías; herramientas comunes cuando el propósito es investigar la reflexión de los maestros en formación.

Encontramos pocos estudios de investigación comparada, y en Colombia pocos estudios de alcance nacional. Realizados como tesis de maestría e incluso de pregrado, se convierten en estudios focalizados o centrados en un solo caso, con muestras muy pequeñas que limitan la magnitud de sus resultados. El enfoque de sistematización de experiencias o evaluación de propuestas prevalece sobre otros, de manera que, escasean los trabajos que desde nuestro contexto aportan lecturas teóricas o epistemológicas sobre el problema de la práctica en los procesos de formación de maestros. 
Tabla 2

Diseños metodológicos

\begin{tabular}{|c|c|c|}
\hline $\begin{array}{l}\text { Enfoque de } \\
\text { investigación }\end{array}$ & $\begin{array}{l}\text { Herramientas de } \\
\text { recolección de datos }\end{array}$ & Ejemplos \\
\hline \multirow{14}{*}{$\begin{array}{l}\text { Cualitativo (estudios } \\
\text { de caso, etnografía, } \\
\text { narrativas, etc.) }\end{array}$} & Relatos autobiográficos & $\begin{array}{l}\text { Barreto, Mateus y Muñoz 2011; Chavarría } \\
\text { y Echavarría, 2014; Páez et al., } 2008\end{array}$ \\
\hline & Autorreflexión & Ajayi, 2011; Daniels et al., 2013; Schulz, 2005 \\
\hline & Grupos reflexivos & Bowman y Herrelko, 2014; Malkani y Allen, 2005 \\
\hline & Diarios reflexivos & $\begin{array}{c}\text { Jiménez, 2010; Rakicioglu-Soylemez y Eroz- } \\
\text { Tuga, 2014; Sagayo y Chacón, } 2006\end{array}$ \\
\hline & Entrevista & $\begin{array}{l}\text { Binks et al., 2009; Göçer, 2013; Herrera y Larios, } \\
\text { 2012; Madlela, 2015; Pérez et al., } 2006\end{array}$ \\
\hline & Encuesta & $\begin{array}{l}\text { Cometta, Domeniconi, et al., 2005; Quintero, } \\
\text { 2008; Quintero y Duque, } 2010\end{array}$ \\
\hline & Observación & $\begin{array}{l}\text { Göçer, 2013; Harrison et al., 2005; Joseph } \\
\text { y Heading, 2010; Mannathoko, } 2013\end{array}$ \\
\hline & Bitácoras & Harrison et al., 2005 \\
\hline & $\begin{array}{l}\text { Acompañamiento a grupos } \\
\text { de prácticas profesionales }\end{array}$ & Harrison et al., 2005; Villa Holguín, 2013 \\
\hline & Escalas Likert & Gallego et al., 2006 \\
\hline & Grupo focal & $\begin{array}{l}\text { López-Vargas y Basto-Torrado, 2010; } \\
\text { Villegas et al., 2015; Yalin UÇar, } 2012\end{array}$ \\
\hline & Portafolio electrónico & Campaña, 2013; Lara et al., 2015 \\
\hline & Estudio de caso & $\begin{array}{l}\text { Rakicioglu-Soylemez y Eroz-Tuga, 2014; } \\
\text { Schrittesser, 2008; Villegas et al., } 2015\end{array}$ \\
\hline & Histórica & Conde y Cortés, 2006 \\
\hline \multirow{2}{*}{ Cuantitativo } & Encuesta & Endeley y Nalova, 2014; Oluwatayo \& Adebule, 2012 \\
\hline & Cuasi experimental & Karami et al., 2013; Koç, 2011 \\
\hline \multirow{2}{*}{ Mixtos } & $\begin{array}{l}\text { Cuestionarios } \\
\text { pretest - postest }\end{array}$ & Busher et al., 2015; Jones, 2009; White Dean y Todd, 2000 \\
\hline & Entrevista semiestructurada & Busher et al., 2015 \\
\hline \multirow{2}{*}{ Otros } & Sistematización reflexiva & Arenas et al., 2015 \\
\hline & Estado del arte & Bolaño, 2014; Lawson et al., 2015 \\
\hline
\end{tabular}


Aunque muchos estudios reconocen que la perspectiva teórica y metodológica de la práctica reflexiva está relacionada con las teorías críticas, las investigaciones sobre este tema no se conducen desde ese enfoque. La mayoría de los trabajos se desarrollan como investigación cualitativa y retoman la voz de alguno de los actores de la práctica. Tal como lo habían identificado Lawson et al (2015), hacen falta estudios que recuperen la pluralidad de las voces involucradas en estos procesos de formación. No encontramos trabajos que mostraran la voz, percepción o significación de los maestros receptores de los estudiantes de práctica, también llamados en algunos contextos "maestros cooperadores"; tampoco se identifican trabajos donde se recupere la voz de los estudiantes que reciben a estos maestros practicantes en sus aulas. Estos dos asuntos demandan más atención de los investigadores, porque nos permitirían tener una lectura más completa de esta importante etapa en el proceso de formación de educadores.

\section{Conclusiones}

La gran cantidad de trabajos identificados para realizar este estado del arte es una evidencia del posicionamiento de la práctica pedagógica como un objeto destacado de las preocupaciones de los formadores de maestros; su inquietud por la efectividad o los efectos de las prácticas en los maestros en formación podría considerarse global y de la agenda presente de estos formadores.

Se ha visibilizado cómo el discurso de la práctica reflexiva es el soporte de la mayoría de las investigaciones sobre la práctica pedagógica - independientemente de su enfoque metodológico-; con él, se sostienen estudios experimentales, comunidades de saber - como la Plataforma Internacional de Práctica Reflexiva-, políticas públicas, entre otros. Todo esto sin preguntarse por la pertinencia de este discurso ni por el papel que desde allí se otorga a los maestros en formación y a los maestros formadores.

En nuestro país la política educativa hoy hace un llamado a las instituciones formadoras para aumentar las prácticas en los programas de formación, incluso se plantea que el cambio debe orientarse desde la práctica reflexiva; sin embargo, hasta ahora este no es el tema de debate en las investigaciones en Colombia. Se hace necesario emprender estudios más globales sobre el problema de la práctica, y sobre los desafíos que se presentan para su implementación, tanto los que son de orden académico y pedagógico como aquellos de orden administrativo.
Los estudios comparados siguen pendientes en la agenda investigativa de las prácticas en nuestro contexto. Trabajamos en investigaciones autorreferenciales, localizadas, que poco conversan con los movimientos teóricos y metodológicos sobre formación de educadores a nivel global; prueba de ello es que el cambio que agencia hoy la política pública en Colombia, en otros países de Latinoamérica, como Argentina o Chile, lleva más de veinte años de implementación. La pluralidad de los diseños metodológicos que aquí se han identificado puede convertirse en motivador de nuevos estudios con mayor alcance en lo referente a contextos, voces y problemáticas, pues como se ha mostrado, el campo de la formación de maestros vive en permanente debate y construcción.

En conclusión, investigar la práctica pedagógica en nuestro país sigue siendo un desafío que va más allá de las sistematizaciones, necesitamos conocer lo que ocurre desde la perspectiva de los múltiples actores que intervienen en el proceso; asimismo, es clave desarrollar nuevos dispositivos de formación que no se agotan en las técnicas narrativas. Los estudios aquí reseñados nos muestran interesantes alternativas.

\section{Referencias}

Ajayi, L. (2011). Teaching alternative licensed literacy teachers to learn from practice: a critical reflection model. Teacher Education Quarterly, 169-189.

Arenas, J., Bermúdez, J. y Fandiño, Y. (2015). Un proceso de sistematización del trabajo de directores de práctica pedagógica: Algunas reflexiones desde la formación docente. Educación y Ciudad, 29, 199-212.

Baquero, P. (2006). Práctica pedagógica, Investigación y Formación de Educadores. Tres concepciones dominantes de la práctica docente. Actualidades Pedagógicas, 49, 9-22. https://doi.org/10.19052/ap.1612

Barragán, D. (2015). El saber práctico: Phrónesis, hermenéutica del quehacer del profesor. Bogotá: Unisalle.

Barreto, J., Mateus, C. y Muñoz, C. (2011). La práctica reflexiva, estrategia para reconstruir el pensar y hacer las prácticas de enseñanza (Tesis de Maestría). Pontificia Universidad Javeriana, Bogotá. Recuperado de https://repository.javeriana.edu.co:8443/bitstream/ handle/10554/1260/BarretoLondonoJazmine2011. pdf? sequence $=1$ \&isAllowed $=y$

Binks, E., Smith, D., Smith, L. y Joshi, R. (2009). Tell me your story: A reflection strategy for preservice teachers. Teacher Education Quartely, 141-156. 
Bolaño, Y. (2014). Estado del arte sobre las investigaciones de prácticas pedagógicas de los maestros en formación en universidades colombianas en el periodo del 2010 al 2013 (Tesis de Maestría). Universidad de San Buenaventura, Bogotá.

Bowman, C. y Herrelko, J. (2014). Bridging theory to practice with classroom rounds. Journal of the Scholarship of Teaching and Learning, 14 (4), 51-66. Recuperado de https://doi.org/10.14434/josotl.v14i4.5185

Busher, H., Gündüz, M., Çakmak, M. y Lawson, T. (2015). Student teachers' views of practicums (teacher training placements) in Turkish and English contexts: a comparative study. Compare: A Journal of Comparative and International Education, 45 (3), 445-466. Recuperado de https://doi.org/10.1080/03057925.2014.930659

Campaña, K. (2013). El portafolio virtual como herramienta para la práctica reflexiva. Valoración de su empleo en el prácticum de magisterio de la Universidad de Navarra (Tesis de Doctorado). Universidad de Navarra. Recuperado de https://www.educacion.gob.es/teseo/ mostrarRef.do?ref=344718

Caner, M. (2010). A blended learning model for teaching practice course. Turkish Online Journal of Distance Education, 11 (3), 77-97. Recuperado de http://dergipark. ulakbim.gov.tr/tojde/article/view/5000102523

Chavarría, D. y Echavarría, P. (2014). El relato de sí: Dispositivo para la construcción de las prácticas pedagógicas (Trabajo de grado). Universidad de Antioquia, Medellín. Recuperado de http://ayura.udea.edu.co:8080/jspui/ bitstream/123456789/1189/1/CA/0653

Choy, S. y San Oo, P. (2012). Reflective thinking and teaching practices: a precursor for incorporating critical thinking into the classroom? International Journal of Instruction, 55 (11), 167-182.

Cometta, A., Bentolila, S., Clavijo, M., Domeniconi, R., y López, Z. (2005). Las innovaciones como prácticas emergentes en el proceso de enseñar. Aproximaciones a su estudio en carreras de formación docente de la Universidad Nacional de San Luis UNSL. En M. Vitarelli (Ed.), Formación docente e investigación: propuestas en desarrollo (pp. 27-50). San Luis: LAE. Recuperado de http://lae.edu.ar/Ediciones/Indice_Libros.htm

Cometta, A., Domeniconi, R., y López, Z. (2005). El área de la praxis como innovación en la formación docente. Significados y prácticas. En M. Vitarelli (Ed.), Formación docente e investigación: propuestas en desarrollo (pp. 78-96). San Luis: LAE. Recuperado de http://lae.edu. ar/Ediciones/Indice_Libros.htm
Conde, N. y Cortés, A. (2006). Reconstrucción del desarrollo de la práctica pedagógica en la Facultad de Educación de la Universidad de La Salle (Trabajo de grado). Universidad de La Salle, Bogotá. Recuperado de http://repository.lasalle.edu.co/bitstream/handle/10185/8102/ T26.08 M729a.pdf?sequence=1

Da Graca, M. y Mizukami, N. (2002). Learning from cases and bridging some "theory-practice" gaps. Annual World Assembly of the International Council on Education for Teaching, 47th, pp. 1-23. Amsterdam.

Da Silva, M. (2005). Constructing the teaching process from inside out: How pre-service teachers make sense of their perceptions of the teaching of the four skills. Teaching English as a Second or Foreign Language, 9 (2), 1-19. Recuperado de http://www.tesl-ej.org/ wordpress/issues/volume9/ej34/ej34a10/

Daniels, E., Pirayoff, R. y Bessant, S. (2013). Using peer observation and collaboration to improve teaching practices. Universal Journal of Educational Research, 1 (3), 268-274. Recuperado de https://doi.org/10.13189/ ujer.2013.010318

Díaz, S., Barreira, C. y Pinheiro, M. (2015). Evaluación del programa Todos a Aprender: resultados de la evaluación de contexto. Revista de Estudios e Investigación en Psicología y Educación, vol. extr (10). https://doi. org/10.17979/reipe.2015.0.10.360

Domingo Roget, À. (2008). La práctica reflexiva en la formación inicial de maestros/as. Evaluación de un modelo. Tesis doctoral dirigida pel Doctor Albert Arbós Bertrán. Universitat Internacional de Catalunya.

Domingo Roget, À. (2013). Práctica reflexiva para docentes. Saarbrücken: Publicia.

Domingo Roget, À. y Gómez, M. (2014). La práctica reflexiva. Bases, modelos e instrumentos. Madrid: Narcea.

Endeley, M. y Nalova, E. (2014). Teaching practice in Cameroon: The effectiveness of the University of Buea model and implications for quality teaching practice in Cameroon: The effectiveness of the University of Buea model and implications for pre-service teacher education. Australian Journal of Teacher Education, 39 (11). Recuperado de https://doi.org/10.14221/ajte.2014v39n11.9

Gallego, R., Pérez, R., Torres, N. y Gallego Torres, A. (2006). El papel de "las prácticas docentes" en la formación inicial de profesores de ciencias. Revista Electrónica de Enseñanza de las Ciencias, 5 (3), 481-504. Recuperado de http://reec.uvigo.es/volumenes/volumen5/ ART6_Vol5_N3.pdf 
García, H. y Martínez, F. (2006). El sentido de la asesoría en la práctica docente (Trabajo de grado). Universidad de La Salle, Bogotá. Recuperado de http://repository.lasalle.edu.co/bitstream/handle/10185/7978/26011031. pdf? sequence $=1$

Garvis, S. (2012). A self-study in teacher education: Learning to teach in higher education after teaching the arts to young children. US-China Education Review $B$, 3, 298-304. Recuperado de http://files.eric.ed.gov/ fulltext/ED532898.pdf

Göçer, A. (2013). Teacher educator's and guidance teachers' evaluation of student teachers' teaching practice (A qualitative study). Croatian Journal of Education, 15 (3), 125-147.

González, M. y Gómez, P. (2014). Conceptualizing and describing teachers' learning of pedagogical concepts. Australian Journal of Teacher Education, 39 (3912). Recuperado de http://ro.ecu.edu.au/ajte/vol39/iss12/2

Gutiérrez, M. y Buitrago, 0. (2009). La formación docente en las prácticas educativas. Una propuesta basada en la investigación. Pereira: Universidad Tecnológica de Pereira.

Harrison, J., Lawson, T. y Wortley, A. (2005). Mentoring the beginning teacher: developing professional autonomy through critical reflection on practice. Reflective Practice, 6 (3), 419-441. Recuperado de https://doi. org/10.1080/14623940500220277

Herrera, G. y Larios, L. (2012). La constitución de subjetividades. Fundamento para pensar la formación del maestro (Tesis de Maestría). Universidad de San Buenaventura, Bogotá.

Isaza, L., Henao, B. y Gómez, M. (2005). Práctica pedagógica: horizonte intelectual y espacio cultural. Medellín: Universidad de Antioquia, Facultad de Educación.

Jiménez, J. (2010). La práctica reflexiva escritural. Una experiencia con docentes de la asignatura práctica pedagógica de una facultad de educación física (Tesis de Maestría). Pontificia Universidad Javeriana, Bogotá. Recuperado de https://repository.javeriana.edu.co/ handle/10554/1241

Jones, A. (2009). Improving theory application among preservice teachers. Juneau.

Joseph, D. y Heading, M. (2010). Putting theory into practice: Moving from student identity to teacher identity. Australian Journal of Teacher Education, 35 (3).

Karami, M., Karami, Z. y Attaran, M. (2013). Integrating problem-based learning with ICT for developing trainee teachers' content knowledge and teaching skill. International Journal of Education and Development Using
Information and Communication Technology (IJEDICT), 9 (1), 36-49. Recuperado de http://ijedict.dec.uwi.edu// viewarticle.php?id=1578

Kennedy, M. (2004). Reform ideals and teachers' practical intentions. Education Policy Analysis Archives, 13 (13). Recuperado de http://epaa.asu.edu/epaa/v12n13/.

Koç, C. (2011). The views of prospective class teachers about peer assessment in teaching practice. Educational Sciences: Theory \& Practice, 11 (4), 1979-1989.

Korthagen, F. (1985). Reflective teaching and preservice teacher education in the Netherlands. Journal of teacher education, 36 (5), 11-15. Recuperado de https://doi. org/10.1177/002248718503600502

Korthagen, F. (1988). The influence of learning orientations on the development of reflective teaching. Teachers' professional learning, 35-50. Falmer Press London.

Korthagen, F. (2010). La práctica, la teoría y la persona en la formación del profesorado. Revista Interuniversitaria de Formación del Profesorado, 68 (24, 2), 83-101.

Korthagen, F., Loughran, J. y Russell, T. (2006). Developing fundamental principles for teacher education programs and practices. Teaching and Teacher Education, 22 (8), 1020-1041. Recuperado de https://doi.org/10.1016/j. tate.2006.04.022

Lara, B., Pereira, M., Alvarado, P. y Muñoz, C. (2015). Reflexión de la práctica pedagógica, a través de un portafolio electrónico, en la formación inicial docente. Conocimiento Educativo, 2, 11-26. Recuperado de http:// www.diyps.catolica.edu.sv/wp-content/uploads/2016 /05/1PortafolioDocCEVol2.pdf

Lawson, T., Çakmak, M., Gündüz, M. y Busher, H. (2015). Research on teaching practicum -a systematic review. European Journal of Teacher Education, 38 (3), 392-407. Recuperado de https://doi.org/10.1080/02619768.2 014.994060

López-Vargas, B. y Basto-Torrado, S. (2010). Desde las teorías implícitas a la docencia como práctica reflexiva. Educación y Educadores, 13 (2), 275-291.

López Gil, M. (2010). Genealogía de la práctica pedagógica para la formación de maestros normalistas. Plumilla Educativa, 7, 84-91. Recuperado de https://issuu.com/ umzl/docs/plumilla_7

Madlela, B. (2015). ICT opportunities and threats in implementing teaching practice programmes. Universal Journal of Educational Research, 3 (6), 351-358. Recuperado de https://doi.org/10.13189/ujer.2015.030601

Malkani, J. y Allen, J. (2005). Cases in teacher education: Beyond reflection into practice. Annual Meeting of the American Educational Research Association, p. 31. 
Montreal. Recuperado de http://citeseerx.ist.psu.edu/ viewdoc/download?doi=10.1.1.963.1925\&rep=rep1 \&type $=$ pdf

Mannathoko, M. (2013). Does teaching practice effectively prepare student-teachers to teach creative and performing arts? The case of Botswana. International Journal of Higher Education, 2 (115), 116-121. Recuperado de https://doi.org/10.5430/ijhe.v2n2p115

Niño, F. (2011). La formación inicial del docente como investigador de su práctica pedagógica. Bogotá: Bonaventuriana.

Oluwatayo, J. y Adebule, S. (2012). Assessment of teaching performance of student-teachers on teaching practice. International Education Studies, 5 (5), 109-115. Recuperado de https://doi.org/10.5539/ies.v5n5p109

Páez, A., Gómez, C. y García, M. (2008). La escritura autobiográfica como estrategia de reflexión de las prácticas pedagógicas: análisis de los discursos de los maestros (Tesis de Maestría). Universidad de La Salle, Bogotá. Recuperado de http://repository.lasalle.edu. co/bitstream/handle/10185/1454/T85.08 P139e. pdf?sequence $=1$

Pérez, Y., Rodríguez, M. y Triana, M. (2006). Lineamientos curriculares que promueven la formación de profesionales reflexivos (Tesis de Maestría). Universidad de La Salle, Bogotá. Recuperado de http://repository.lasalle. edu.co/handle/10185/1391

Perrenoud, P. (2004). Desarrollar la práctica reflexiva en el oficio de enseñar. Barcelona: Grao. Recuperado de www.grao.com

Quintero, M. (2008). Propuesta metodológica para la enseñanza de la Práctica Docente en la universidad. Uni-Pluri/versidad, 8 (1), 1-13. Recuperado de https:// aprendeenlinea.udea.edu.co/revistas/index.php/unip/ article/viewFile/7205/6660

Quintero, M. y Duque, S. (2010). Modelo de autoevaluación de la Práctica Pedagógica para los programas de licenciatura de Facultades de Educación. Uni-Pluri/ versidad, 10 (1), 1-11.

Rakicioglu-Soylemez, A. y Eroz-Tuga, B. (2014). Mentoring expectations and experiences of prospective and cooperating teachers during practice teaching. Australian Journal of Teacher Education, 39 (10), 145-168. Recuperado de https://doi.org/10.14221/ajte.2014v39n10.10

Rodríguez, A., Esteban, R., Aranda, R., Blanchard, M., Domínguez, C., González, P., ... Messina, C. (2011). Coaching reflexivo entre iguales en el Practicum de la formación de maestros. Revista de Educación, 355, 355-379. Recuperado de https://doi. org/10-4438/1988-592X-RE-2011-355-02
Sagayo, Z. y Chacón, M. (2006). Las prácticas profesionales en la formación docente: hacia un nuevo diario de ruta. Educere, 10 (32), 55-66. Recuperado de http://www. redalyc.org/articulo.oa?id=35603209

Saker García, J. (2014). Práctica pedagógica investigativa en las Escuelas Normales Superiores: contexto y pertinencia de la calidad educativa. Educación y Humanismo, 16 (26), 83-103. Recuperado de http://publicaciones. unisimonbolivar.edu.co/rdigital/educacion/index. php/educacion

Schrittesser, I. (2008). The role of case study in teacher education: an attempt to bridge the gap between action and reflection. Global Education Review, 1 (4), 187-194. Recuperado de http://ger.mercy.edu/index.php/ger/ article/view/108

Schulz, R. (2005). The Practicum: More than practice. Canadian Journal of Education/Revue Canadienne de L'éducation, 28 (1/2), 147-167. Recuperado de https:// doi.org/10.2307/1602158

Schön, D. (1983). The reflective practitioner: how professionals think in action. New York: Basic Books.

Schön, D. (1987). Educating the reflective practitioner: Toward a new design for teaching and learning in the professions. San Francisco: Jossey Bass.

Torres, M. y Mercado, M. (2004). Living the praxis of teacher education through teacher research. Scholar-Practitioner Quarterly, 2 (2), 59-73. Recuperado de http://files. eric.ed.gov/fulltext/EJ796112.pdf

Villa Holguín, E. (2013). La praxis como búsqueda ética en los escenarios de las prácticas formativas e investigativas. Itinerario Educativo, 27 (62), 255-268. Recuperado de http://revistas.usb.edu.co/index.php/Itinerario/ article/view/1497/1268

Villalobos, J. y de Cabrera, C. (2009). Los docentes y su necesidad de ejercer una práctica reflexiva. Revista de Teoría y Didáctica de las Ciencias Sociales, 14, 139-166.

Villegas, A., Londoño, L., Cárdenas, M. y Mosquera, Y. (2015). Análisis de la práctica pedagógica en la Licenciatura en educación básica con énfasis en Ciencias naturales y Educación ambiental: con miras al fortalecimiento del vínculo universidad - centro de práctica. Universidad de Antioquia. Recuperado de http://ayura.udea. edu.co:8080/jspui/bitstream/123456789/1859/1/ JE0977.pdf

Vitarelli, M. (2005). La práctica docente y la realidad del aula. Una experiencia de formación en investigación educativa. En M. Vitarelli (Ed.), Formación docente y prácticas pedagógicas (pp. 97-127). San Luis: LAE. Recuperado de http://lae.edu.ar/Ediciones/Indice_Libros.htm 
White Dean, E. y Todd, G. (2000). Changing teaching practices by empowering teachers with research knowledge. Annual Meeting of the Mid-South Educational Research Association, p. 33. Bowling Green.
Yalin UÇar, M. (2012). A case study of how teaching practice process takes place. Educational Sciences: Theory \& Practice, 12 (4), 2654-2659. Recuperado de http://www.kuyeb.com/pdf/en/ca73f201777c71e63c98a581938eaa1ecaren.pdf 